\title{
Interacting fermions in two dimensions: beyond the perturbation theory
}

\author{
Suhas Gangadharaiah ${ }^{1}$, Dmitrii L. Maslov ${ }^{1 *, 2}$, Andrey V. Chubukov ${ }^{3}$, and Leonid I. Glazman ${ }^{4}$ \\ ${ }^{1}$ Department of Physics, University of Florida, P. O. Box 118440, Gainesville, FL 32611-8440 \\ ${ }^{2}$ Abdus Salam International Center for Theoretical Physics, 11 Strada Costiera, 34014 Trieste, Italy \\ ${ }^{3}$ Department of Physics, University of Maryland, College Park, MD 20742-4111 \\ ${ }^{4}$ Theoretical Physics Institute, University of Minnesota, Minneapolis, MN 55455
}

(Dated: August 26, 2018)

\begin{abstract}
We consider a system of 2D fermions with short-range interaction. A straightforward perturbation theory is shown to be ill-defined even for an infinitesimally weak interaction, as the perturbative series for the self-energy diverges near the mass shell. We show that the divergences result from the interaction of fermions with the zero-sound collective mode. By re-summing the most divergent diagrams, we obtain a closed form of the self-energy near the mass shell. The spectral function exhibits a threshold feature at the onset of the emission of the zero-sound waves. We also show that the interaction with the zero sound does not affect a non-analytic, $T^{2}$-part of the specific heat.
\end{abstract}

PACS numbers: 71.10.Ay,71.10.Pm

The Landau Fermi-liquid (FL) theory states that the low-energy properties of an interacting fermion system are similar to those of an ideal Fermi gas [1]. A necessary (but not sufficient) condition for the validity of this theory is that various characteristic properties (thermodynamic parameters, quasi-particle lifetime, etc.) can be expressed via regular perturbative expansions in the interaction. Back in the $50 \mathrm{~s}$, it was shown to be the case for a model of 3D fermions with both short- and long-range (Coulomb) repulsion. Soon thereafter, it was reazlied that the perturbation theory is singular in 1D and, as a result, the FL is destroyed. The case of $D=2$ had been the subject of an active and fairly recent discussion, with a number of proposals, most notably by Anderson [2], for the breakdown of the FL in 2D. Although the prevailing opinion currently is that the FL is stable in $2 \mathrm{D}$ for sufficiently short-range (including Coulomb) interactions, a full description of the FL in 2D is still lacking.

One of the problems in describing a 2D interacting system is that, in a contrast to the $3 \mathrm{D}$ case, a naive perturbative expansion in the interaction is singular. Namely, for a linearized single-particle spectrum, the imaginary part of the self-energy diverges on the mass shell. The divergence is logarithmic to second order in the interaction [3, 4, 5, 6] but, as we will show in this paper, it is amplified to a power-law, starting from the third order. This mass-shell singularity in 2D is a weaker form of the "infrared catastrophe" in 1D. There, an on-shell fermion can emit an infinite number of soft bosons-quanta of charge- and spin-density fluctuations [], which gives rise to a power-law divergence of the self-energy on the massshell. In 2D, this mechanism is weakened but not completely eliminated (the "memory" about the 1D infrared catastrophe is erased completely only for $D>2$ ). At a first glance, the breakdown of the perturbation theory confirms the conjecture that the FL is destroyed in $2 \mathrm{D}$ [2]. However, all it really means is that in order to obtain physically meaningful results the perturbation the- ory must be re-summed even for an arbitrarily weak interaction [3, 5].

In this Letter, we report the results of an asymptotically exact re-summation of the divergent perturbation theory for $D=2$. In addition to obtaining a closed and divergence-free form of the self-energy to all orders in the interaction, this procedure also allows one to identify the nature of the singularities in a perturbation theory as originating from the interaction between the fermions and the zero-sound (ZS) collective mode. At any finite order of the perturbation theory, the collective mode coincides with the upper edge of the particle-hole $(\mathrm{PH})$ continuum. In $2 \mathrm{D}$, this degeneracy is strong enough to generate the divergences in the self-energy. Once perturbations are summed up to all orders, the zero-sound mode splits off from the continuum, and the power-law divergences disappear. The remaining log-divergences are eliminated by restoring the finite curvature of the spectrum near the Fermi surface [5, 6]. Thus the FL survives. However, the resulting self-energy contains several non-perturbative features that signal a deviation from the standard FL behavior. First, the imaginary part of a non-perturbative, ZS contribution to the selfenergy, $\Sigma_{\mathrm{ZS}}(\omega, k)$, is a non-monotonic function of the "distance" to the mass-shell, $\Delta \equiv \omega-\epsilon_{k}$, and exhibits an anomaly at the threshold for emission of ZS waves. This anomaly gives rise to a non-Lorentzian shape of the spectral function. Second, as the theory is regularized by the difference in the ZS and Fermi velocities, which is small for a weak interaction, $\Sigma_{\mathrm{ZS}}$ is strongly enhanced near the mass shell. On the mass shell, $\operatorname{Re} \Sigma_{Z S}$ is of the same $-U^{2} \omega|\omega|$-order, as the perturbative contribution. It has been argued that the non-analytic, perturbative self-energy $\operatorname{Re} \Sigma \propto U^{2} \omega|\omega|$ gives rise to a non-analytic, $T^{2}$-term in the specific heat $C(T)[\underline{6},[, 8,9]$. Therefore, it becomes an issue whether the non-perturbative part of the self-energy also contributes to the $T^{2}$-term in $C(T)$. We show that this is not the case, as the thermodynamic 
a)

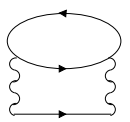

b)
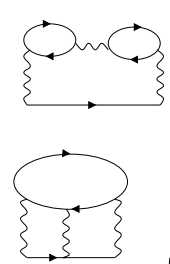
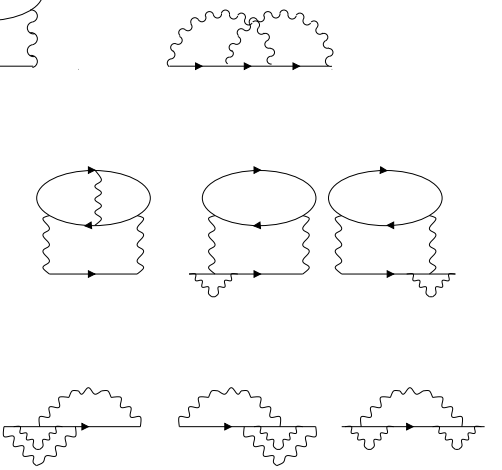

FIG. 1: Maximally divergent self-energy diagrams to second (a) and third (b) orders in the interaction.

potential is not affected by the mass-shell singularities.

In what follows, we present the results and outline the main steps. Detailed calculations can be found in the extended version of this communication [10].

To understand the origin of the mass-shell singularity in arbitrary dimension $D$, consider the self-energy to second order in the interaction (first two diagrams in Fig 1), and focus on small-angle scattering of fermions with almost parallel momenta (forward scattering), as shown in Fig. [2].

The imaginary part of the retarded self-energy on the mass shell is given by (for $\omega>0$ )

$$
\begin{aligned}
\operatorname{Im} \Sigma_{\mathrm{F}}\left(\omega=\epsilon_{k}\right) \propto & u_{0}^{2} \int_{-\omega}^{0} d \Omega \int d Q Q^{D-1} \int d O_{D} \\
& \times \delta\left(\Omega+v_{F} Q \cos \vartheta\right) \operatorname{Im} \Pi(\Omega, Q),(1)
\end{aligned}
$$

where $u_{0} \equiv U(0) m / 2 \pi$ is the dimensionless coupling constant, $\Pi(\Omega, Q)$ is the polarization bubble, and $d O_{1}=$ $\delta(\vartheta) d \vartheta, d O_{2}=d \vartheta, d O_{3}=\sin \vartheta d \vartheta . \quad \operatorname{Im} \Pi(\Omega, Q)$ is the probability amplitude to generate a $\mathrm{PH}$ pair of frequency $\Omega$ and momentum $Q$. For $D=1$, the $\mathrm{PH}$ continuum consists of a single line $\Omega=v_{F} Q$ and $\operatorname{Im} \Pi(\Omega, Q) \propto$ $\delta\left(\Omega-v_{F} Q\right)$. A product of two $\delta$-functions in (11) yields a non-integrable singularity, which is the origin of an infrared catastrophe [7]. For $D>1$, the $\mathrm{PH}$ continuum is a broad band specified by $|\Omega| \leq v_{F} Q$, and the fermion spectral function is softened by angle-averaging. For $D=3$, the resulting self-energy is finite on the mass shell. However, for $D=2$ the angle-averaged fermion spectral function still has a square- root singularity: $\int d \theta \delta\left(\Omega-v_{F} Q \cos \vartheta\right) \propto\left(v_{F} Q-|\Omega|\right)^{-1 / 2}$ for $v_{F} Q>|\Omega|$, whereas $\operatorname{Im} \Pi(\Omega, Q) \propto \Omega /\left(v_{F} Q-|\Omega|\right)^{1 / 2}$ has another square-root singularity at the continuum boundary. Merging of the two square-root singularities results in a logarithmic divergence of $\operatorname{Im} \Sigma$ on the mass shell. Near the mass shell $(|\Delta| \ll|\omega|), \operatorname{Im} \Sigma \propto \ln |\Delta|$.

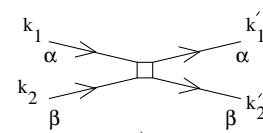

a)

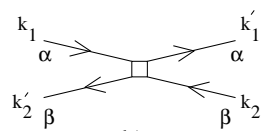

b)

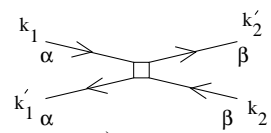

c)
FIG. 2: Forward(a) and backscattering $(\mathrm{b}+\mathrm{c})$ scattering processes.

Higher-order diagrams contain higher powers of divergent $\mathrm{PH}$ bubbles; these bubbles are either explicit, as in the ring diagrams of Fig. 11 or generated by integrating out the fermionic energy and momenta. One can verify that the square-root singularities accumulate, and the higher-order diagrams for the selfenergy diverge as powers of $\Delta^{-1}$. At the n-th order, $\operatorname{Im} \Sigma_{F} \propto u_{0}^{n} \omega^{2}(\omega / \Delta)^{n / 2-1} \theta(\omega / \Delta)$, where $\theta(x)$ is the step-function. By Kramers-Kronig relation, these singularities generate similar power-law divergences in $\operatorname{Re} \Sigma_{F}$. Hence, to find the self-energy in $D=2$ one has to re-sum the perturbative series even for an infinitesimally small $U$.

This summation can be carried out explicitly for small $U$ by collecting diagrams with the maximum number of polarization bubbles at each order (to third order, such diagrams are shown in Fig. (1). The result is

$$
\begin{aligned}
& \Sigma_{F}(p)=\frac{1}{2} \int_{q} G(p-q)\left[4 U(0)-2 U^{2}(0) \Pi(q)\right. \\
& \left.+\quad \frac{U(0)}{1-U(0) \Pi(q)}-\frac{3 U(0)}{1+U(0) \Pi(q)}\right]
\end{aligned}
$$

where $q \equiv(\mathbf{Q}, \Omega)$ and $\int_{q} \ldots \equiv T \sum_{\Omega_{m}} \int d^{2} q /(2 \pi)^{2} \ldots$ The last two terms in Eq. (2) correspond to the interaction in the charge and spin channels, respectively; the first two terms contain unaccounted parts of first- and second-order diagrams. All terms in Eq. (2) have perturbative contributions from the $\mathrm{PH}$ continuum. In addition, the charge part contains a non-perturbative contribution from the ZS pole in the charge-channel propagator. Near the pole, $[1-U(0) \Pi(q)]^{-1} \propto u_{0}^{2} Q^{2} /\left(\Omega^{2}-\right.$ $\left.c^{2} Q^{2}\right)$, where $c=v_{F}\left(1+u_{0}^{2} / 2+\ldots\right)$ is the $\mathrm{ZS}$ velocity. Notice that the quanta of ZS are not free bosons: the residue of the pole vanishes at $Q=0$, as it is required by the translational invariance of the system. Substituting the ZS propagator into Eq. (2), we obtain for the corresponding contribution to the self-energy

$$
\begin{aligned}
& \operatorname{Re} \Sigma_{\mathrm{ZS}}=\frac{u_{0}^{2} \omega^{2}}{8 E_{F}} F_{R}\left(\frac{\Delta}{\Delta^{*}}\right) \\
& \operatorname{Im} \Sigma_{\mathrm{ZS}}=\frac{u_{0}^{2} \omega^{2}}{4 \pi E_{F}} F_{I}\left(\frac{\Delta}{\Delta^{*}}\right),
\end{aligned}
$$

where $\Delta^{*} \equiv u_{0}^{2} \omega / 2$, and functions $F_{R, I}$ are the real and 


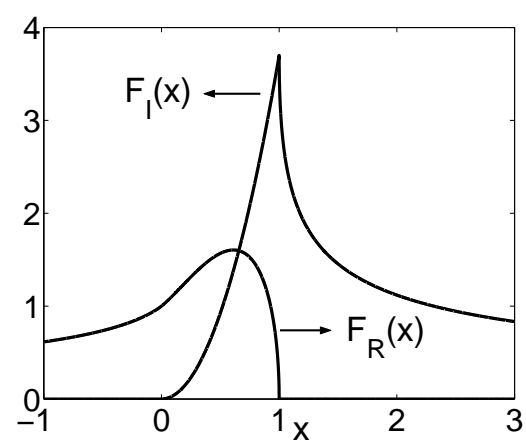

FIG. 3: Scaling functions $F_{I}(x)$ and $F_{R}(x)$

imaginary parts of

$$
F(x)=\left(1+\frac{3}{2} x\right) \sqrt{1-x}+\frac{3}{2} x^{2} \ln \frac{1+\sqrt{1-x}}{\sqrt{-x}},
$$

correspondingly. Plots of $F_{R, I}$ are presented in Fig. 3. Notice that $F_{I}(x<0)=0$ and $F_{R}(x>1)=0$.

For large $x$, i.e., far away from the mass shell, $F_{I}(x) \propto$ $1 / \sqrt{x}$, and $\operatorname{Im} \Sigma_{\mathrm{ZS}} \propto u_{0}^{3} \omega^{5 / 2} / \sqrt{\Delta}$. This behavior reproduces the result of the perturbation theory. Expanding further in $1 / x$, one obtains higher powers of $1 / \Delta$. However, we see from (44) that the emerging singularity at $\Delta=0$ is actually cut off at $x \simeq 1$, i.e., at $\Delta \simeq \Delta^{*}$. For small $x, F_{I}(x)=3 \pi^{2} x^{2} / 8$, and $\operatorname{Im} \Sigma_{\mathrm{ZS}} \propto \Delta^{2}$ for $\Delta \ll \Delta^{*}$. At $\Delta=0, \operatorname{Im} \Sigma_{\mathrm{ZS}}$ vanishes. Vanishing of $\operatorname{Im} \Sigma_{Z S}$ on the mass shell is a Cherenkov-type effect: because the zero-sound velocity $c>v_{F}$, an on-shell fermion cannot emit a ZS boson. The $\Delta^{2}$-behavior of $\operatorname{Im} \Sigma_{\mathrm{ZS}}$ in between $\Delta=0$ and $\Delta=\Delta^{*}$ tracks an increase of the phase space available for the emission of ZS bosons. We emphasize that as $\Delta^{*} \propto u_{0}^{2}$, the crossover at $\Delta \simeq \Delta^{*}$ could have not been obtained within the perturbation theory. Near $\Delta=\Delta^{*}$, the derivative of $\operatorname{Im} \Sigma(\Delta)$ diverges: $\operatorname{Im} \Sigma_{I}(x) \propto 1 / \sqrt{\Delta-\Delta^{*}}$. The physical meaning of scale $\Delta^{*}$ can be understood by noticing that a Cherenkov-type constraint restricts the frequencies of emitted bosons to the range $0<\Omega<\left(\Delta / \Delta^{*}\right) \omega$ (for $\omega>0$ ). This constraint is relevant for $\Delta<\Delta^{*}$; for larger $\Delta$, emission of bosons with frequencies in the entire interval, allowed by the Pauli principle $(0<\Omega<\omega)$ is possible.

The real part of the self-energy varies slowly near the mass shell as $F_{R}(x \ll 1)=1+x$. Right on the mass shell, $\operatorname{Re} \Sigma_{\mathrm{ZS}} \propto u_{0}^{2} \omega^{2}$. Notice that $\operatorname{Re} \Sigma_{\mathrm{ZS}}$ is of order $u_{0}^{2}$, although it is obtained by summing up terms of order $u_{0}^{3}$ and higher. This enhancement is due to a nonperturbative cut-off of the mass-shell divergences in the perturbation theory.

The remaining contribution to the forward-scattering part of the self-energy comes from the $\mathrm{PH}$ continuum. It contains a logarithmic divergence to second order and also power-law divergences to third and higher orders, as within the perturbation theory the interaction with the continuum is indistinguishable from the interaction with the zero sound. The logarithmic divergence in the selfenergy is cut off by a finite curvature of the spectrum on a scale $\Delta \simeq \omega^{2} / W$, where $W \simeq E_{F}$ is the bandwidth. The power-law divergences in the $\mathrm{PH}$ contribution are cut at the same scale $\Delta^{*}$ as in the $\Sigma_{\mathrm{ZS}}$, this time because an increase of $U(0) \operatorname{Im} \Pi$ near the boundary of the $\mathrm{PH}$ continuum reduces the effective interaction for $\Delta<\Delta^{*}$. However, contrary to the zero-sound contribution, $\Sigma_{\mathrm{PH}}$ is smooth at $\Delta \simeq \Delta^{*}$ as no Cherenkov-type condition is involved. Near the mass shell, $\Sigma_{\mathrm{PH}}$ reduces to

$$
\begin{gathered}
\operatorname{Re} \Sigma_{\mathrm{PH}}=-\frac{u_{0}^{2}|\omega| \Delta}{4 E_{F}} \\
\operatorname{Im} \Sigma_{\mathrm{PH}}=\frac{u_{0}^{2} \omega^{2}}{4 \pi E_{F}} \begin{cases}\left|\ln u_{0}^{2}\right| & \text { for }|\omega| \ll \omega_{c} ; \\
\left|\ln \left(|\omega| / E_{F}\right)\right| & \text { for }|\omega| \gg \omega_{c},\end{cases}
\end{gathered}
$$

where $\omega_{c} \equiv u_{0}^{2} E_{F}$.

The sum $\Sigma_{\mathrm{ZS}}+\Sigma_{\mathrm{PH}}$ is the total contribution to the selfenergy from forward scattering. Another contribution to the self-energy comes from the processes in which the fermions move initially in almost opposite directions, and then either scatter by small angle (Fig. 20) or backscatter (Fig. 2.). We refer to both these processes as "backscattering". The backscattering part of the self-energy, $\Sigma_{\mathrm{B}}$, is regular on the mass shell and, for weak interaction, needs to be evaluated only to second order [6]. Taking the result for $\Sigma_{\mathrm{B}}$ from Ref. [6] and adding it up with the results for $\Sigma_{\mathrm{ZS}}$ and $\Sigma_{\mathrm{PH}}$, we obtain for the total selfenergy on the mass shell

$$
\begin{aligned}
\operatorname{Re} \Sigma(\omega) & =\frac{\omega|\omega|}{8 E_{F}}\left(u_{0}^{2}-\left[u_{0}^{2}+u_{2 k_{F}}^{2}-u_{0} u_{2 k_{F}}\right]\right) \\
& =\frac{\omega|\omega|}{8 E_{F}} u_{2 k_{F}}\left(u_{0}-u_{2 k_{F}}\right) \\
\operatorname{Im} \Sigma(\omega) & =\frac{u_{0}^{2}}{2 \pi} \frac{\omega^{2}}{E_{F}} \ln \frac{E_{F}}{|\omega|} \Phi\left(\frac{|\omega|}{u_{\max }^{2} E_{F}}\right)
\end{aligned}
$$

where $u_{2 k_{F}} \equiv m U\left(2 k_{F}\right) / 2 \pi$ and $u_{\max } \equiv \max \left\{u_{0}, u_{2 k_{F}}\right\}$. The scaling function $\Phi(x)$ approaches the constant values of $1(1 / 2)+u_{2 k_{F}}\left(u_{2 k_{F}}-u_{0}\right) /\left(2 u_{0}\right)$, for $x \rightarrow \infty$ and $x \rightarrow 0$, correspondingly.

The first term in the first line of Eq. (5a is the non-perturbative contribution from forward scattering, whereas the term in square brackets is the backscattering contribution. We see that near the mass shell both contributions are of the same order, i.e., the interaction with the zero-sound collective mode modifies significantly the perturbative result for the self-energy. For contact interaction $\left(u_{0}=u_{2 k_{F}}\right)$, the zero-sound contribution cancels out with the backscattering one, so that the net $\operatorname{Re} \Sigma$ vanishes at the mass shell. At the same time, the effect of the interaction with ZS on the on-shell $\operatorname{Im} \Sigma$ is rather benign: all one has is a smooth crossover function interpolating between two limiting values of the prefactor in 


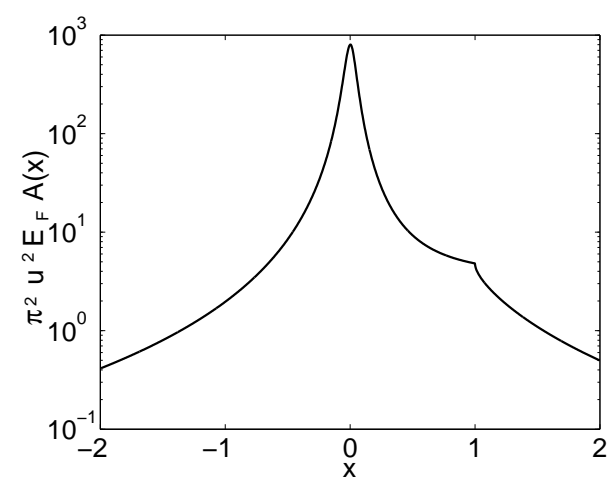

FIG. 4: A log-plot of the spectral function $A(\omega, k)$ as a function of $x=2\left(\omega-\epsilon_{k}\right) / u^{2} \omega$ for fixed $\omega . L_{\omega}=2$ and $\gamma=0.1$ A kink at $x=1$ is a signature of the interaction with the zero-sound mode.

a familiar $\omega^{2} \ln |\omega|$-dependence. This is the consequence of the fact that the non-perturbative, ZS contribution to $\operatorname{Im} \Sigma$ vanishes on the mass shell.

At the same time, away from the mass shell, the full self-energy and the the spectral function $A(\omega, k)=$ $(-1 / \pi) \operatorname{Im} G(\omega, k)$ are affected by the non-monotonic variation of $\operatorname{Im} \Sigma_{\mathrm{ZS}}$ with $\Delta$ near a threshold for emission of ZS bosons. We consider a setup, when $\omega$ is fixed and the spectral function is measured as a function of $k$. This is equivalent to varying $x \equiv \Delta / \Delta^{*}$ at fixed $\omega$. Near the mass shell, the spectral function consists of two terms $A(x)=A_{\mathrm{qp}}(x)+A_{\mathrm{ZS}}(x)$, where

$$
A_{\mathrm{qp}}(x)=\frac{1}{\pi^{2} u^{2} E_{F}} \frac{L_{\omega}}{x^{2}+\gamma^{2}}
$$

is a quasi-particle contribution and

$$
A_{\mathrm{ZS}}=\frac{F_{I}(x)}{\pi^{2} u^{2} E_{F}} \frac{x^{2}-\gamma^{2}}{\left(x^{2}+\gamma^{2}\right)^{2}}
$$

is a ZS contribution. We introduced $L_{\omega} \equiv \ln \left(E_{F} / u^{2}|\omega|\right)$ and $\gamma=|\omega| L_{\omega} /\left(2 \pi E_{F}\right)$, and assumed for simplicity that $u_{0} \equiv u_{2 k_{F}} \equiv u$. The ZS contribution $A_{\mathrm{ZS}}(x)$ has a sharp maximum at $x=1$, where function $F_{I}(x)$ in Fig. 3 has a peak. This maximum gives rise to a kink in total $A(x)$ (see Fig. (4). A similar consideration shows that the kink is present also for the Coulomb interaction. This kink should be detectable in photoemission experiments on layered materials and in momentum-conserving tunneling between parallel layers of 2D electron gases [12].

Finally, we consider an issue whether non-perturbative effects, discussed above, are relevant for the specific heat of two-dimensional fermions. We argue that they are not. The simplest way to see this is to use the definition, $C(T)=-T \partial^{2} \Xi / \partial T^{2}$, and expand the thermodynamic potential $\Xi$ in powers of $U$. The thermodynamic potential can be evaluated in the Matsubara formalism, in which the polarization operator $\Pi_{m}=$ $-(m / 2 \pi)\left[1-\left|\Omega_{m}\right| / \sqrt{\left(v_{F} Q\right)^{2}+\Omega_{m}^{2}}\right]$ is regular at any $\Omega_{m}$ and $Q$. As a result, the Matsubara series for $\Xi$ converges for a weak, short-range interaction, and there is no need for re-summation of the perturbation theory for $\Xi$. Evaluating $\Xi$ to second-order in $U$ yields

$$
\delta C(T)=-\left(u_{0}^{2}+u_{2 k_{F}}^{2}-u_{0} u_{2 k_{F}}\right) \frac{3 m \zeta(3)}{\pi} \frac{T^{2}}{E_{F}} .
$$

This result coincides with $C(T)$ obtained in Ref. [6] by finding the perturbative, backscattering part of the selfenergy first and then using the relation between $C(T)$ and $\Sigma$ [1]. The agreement between the two results shows that non-parturbative, forward-scattering self-energy does not affect $C(T)$.

For completeness, we also verified explicitly that there is no contribution to $C(T)$ from forward scattering. To this end, one can use the relation between $C(T)$ and the Green's function [1] which, to first order in $\left(\omega-\epsilon_{k}\right)^{-1} \Sigma(\omega, k)$, reads

$$
\begin{aligned}
& \delta C(T)=\frac{m T}{\pi} \frac{\partial}{\partial T}\left[\frac{1}{T} \int_{-\infty}^{\infty} d \epsilon_{k} \int_{-\infty}^{\infty} d \omega \omega \frac{\partial n_{0}}{\partial \omega}\right. \\
& \left.\times\left\{\delta\left(\omega-\epsilon_{k}\right) \operatorname{Re} \Sigma^{R}(\omega, k)-\frac{1}{\pi} \mathcal{P} \frac{1}{\omega-\epsilon_{k}} \operatorname{Im} \Sigma^{R}(\omega, k)\right\}\right] .
\end{aligned}
$$

Here $n_{0}(\omega)$ is the Fermi function. It is crucial that both $\operatorname{Re} \Sigma^{R}(\omega, k)$ and $\operatorname{Im} \Sigma^{R}(\omega, k)$ are present in Eq. (9). The forward scattering self energy, $\Sigma_{\mathrm{F}}=\Sigma_{\mathrm{ZS}}+\Sigma_{\mathrm{PH}}$, depends on $k$ due to the presence of a non-perturbative scale $\Delta^{*}$, and therefore $\operatorname{Im} \Sigma_{\mathrm{F}}$ yields a finite contribution to $C(T)$. Substituting the results for $\Sigma_{\mathrm{ZS}}$ and $\Sigma_{\mathrm{PH}}$ into Eq. (9), we find that the forward scattering contributions to the specific heat from $\operatorname{Re} \Sigma_{\mathrm{F}}$ and $\operatorname{Im} \Sigma_{\mathrm{F}}$ cancel each other; hence, there is no contribution to $C(T)$ from forward scattering.

We acknowledge stimulating discussions with I. Aleiner, B. Altshuler, A. Andreev, W. Metzner, A. Millis, and C. Pepin. The research has been supported by NSF DMR 0240238 and Condensed Matter Theory Center at UMD (A. V. Ch.), NSF DMR-0308377 (D. L. M.), and NSF DMR-0237296 (L. I. G.).

[*] Permanent address.

[1] A. A. Abrikosov, L. P. Gorkov, and I. E. Dzyaloshinski, Methods of quantum field theory in statistical physics, (Dover Publications, New York, 1963).

[2] P. W. Anderson, Phys. Rev. Lett. 65, 2306 (1990).

[3] C. Castellani, C. Di Castro, and W. Metzner, Phys. Rev. Lett. 72, 316 (1994).

[4] H. Fukuyama and M. Ogata, J. Phys. Soc. Jpn. 63, 3923 (1995).

[5] C. Halboth and W. Metzner, Phys. Rev. B 57, 8873 (1998). 
[6] a) A. V. Chubukov and D. L. Maslov, Phys. Rev. B 68, 155113 (2003); b) ibid. 69, 121102 (2004).

[7] Yu. A. Bychkov, L. P. Gor'kov, and I. E. Dzyaloshisnkii, Zh. Eksp. Teor. Fiz. 50, 738 (1966) [Sov. Phys. JETP 23, 489].

[8] D. Coffey and K. S. Bedell, Phys. Rev. Lett. 71, 1043 (1993).

[9] V. M. Galitski and S. Das Sarma, Phys. Rev. B 70, 035111 (2004).

[10] A. V. Chubukov, D. L. Maslov, S. Gangadharaiah, and
L. I. Glazman, cond-mat/0412283

[11] In principle, there is no guarantee that Eq. (9) works for non-linear terms in $C(T)$. However, it can be verified by a direct comparison with the general Luttinger-Ward formula that the $T^{2}$-term is correctly described by (9) if one uses the $T$-dependent self-energy rather than the zero-temperature one.

[12] see S. Q. Murphy, J. P. Eisenstein, L. N. Pfeiffer, and K. W. West, Phys. Rev. B 52, 14825-14828 (1995) and references therein. 